\title{
Investigation of the influence of radial grooves on the flow in an eccentrically deposited annulus using CFD numerical simulation
}

\author{
Marian Bojko ${ }^{1, a}$, Milada Kozubkova ${ }^{1}$, Michal Kozdera ${ }^{1}$, Ondřej Zavila ${ }^{2}$ \\ ${ }^{1}$ Department of Hydromechanics and Hydraulic Equipment, Faculty of Mechanical Engineering, VŠB-Technical University \\ of Ostrava, 17. listopadu 15, Ostrava, Czech Republic \\ ${ }^{2}$ Department of Fire Protection, Faculty of Safety Engineering, VŠB-Technical University of Ostrava, 17. listopadu 15, \\ Ostrava, Czech Republic
}

\begin{abstract}
This paper evaluates the influence of radial grooves in the eccentrically deposited annulus using by mathematical modeling of fluid flow through each grooves. The inner cylinder (rotor) is eccentrically deposited and its movement is composed of two movements (rotation and precession). The outer cylinder is stationary and has radial grooves. In the first phase is defined a mathematical model of the flow, which is then applied to the 3D model of narrow gap with radial grooves. In this paper are present several variants of the computational domain with regard to the number of radial grooves. Based on the results of numerical simulations are evaluated basic variables such as pressure and velocity. There are also evaluated radial force $\left(F_{\mathrm{r}}\right)$ and axial force $\left(F_{a}\right)$ acting on the rotor for different boundary conditions (different speeds of rotation and precession). Subsequently, the pressure gradients $(\Delta \mathrm{p})$ are evaluated between the radial grooves for different computational domain. Results are compared to variant of narrow gap without radial grooves. Numerical simulation is realized in the program system ANSYS Fluent.
\end{abstract}

\section{Introduction}

Hydrodynamic machines consist of a system of hydrodynamic elements, for example bearings, impellers, seals and sealing gaps that include narrow gaps where the flow losses are occurred [1]. The aim of this paper is the solution flow in the narrow gap representing the sealing gap with radial grooves. The fluid flow of the sealing gap is solved numerically through CFD analysis by software ANSYS Fluent. In this study, a small gap is represented by annular, when the inner cylinder (rotor) is eccentrically deposited within the outer cylinder (stator). Rotor carried rotational movement around its own axis and processional movement in a circle. The first phase was defined mathematical model of flow in the narrow gap as a system of partial differential equations (continuity equation and Navier-Stokes equations) for laminar flow of a real fluid. Rotation and precession of the rotor was defined using user-defined functions and implemented into a mathematical model. The final mathematical model was then applied to the $3 \mathrm{D}$ model annular gap with radial grooves. For solution of the compiled mathematical model was used ANSYS Fluent software program. Numerical simulations were solved different variations for different angular velocity of rotation and precession of the rotor in geometry with four radial grooves. Basic dimensions of the narrow gaps are

\footnotetext{
${ }^{\mathrm{a}}$ Corresponding author: marian.bojko@vsb.cz
}

shown in Figure 1. The arrangement and dimensions of radial grooves in longitudinal section are illustrated in Figure 2. From Figure 2 it is also evident inlet of fluid into the computational domain.

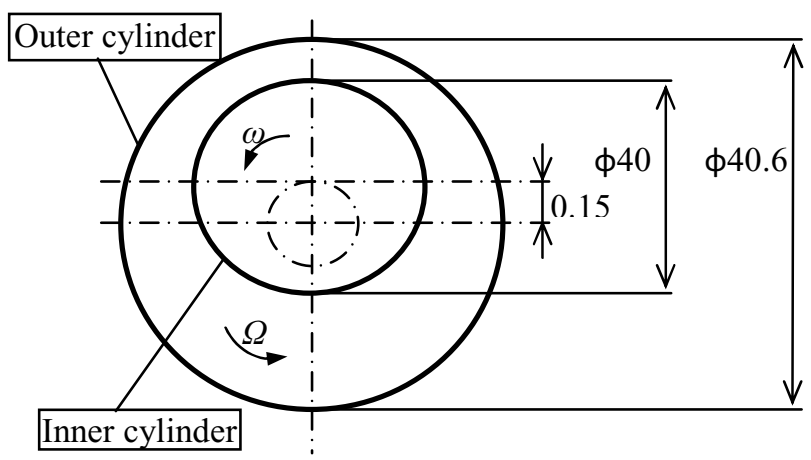

Figure 1. Dimensions of narrow gap

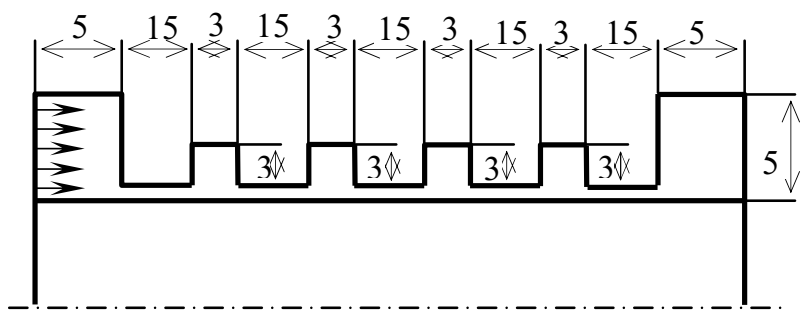

Figure 2. Longitudinal section through the 3D model with four radial grooves 
The final 3D model of the computational domain with shown inlet and radial grooves is shown in the Figure 3. The outlet of fluid is on the opposite side as the fluid inlet. Then the fluid enters into the inlet pocket, then passes through a narrow gap in the first radial groove and once again through narrow gap to the next radial groove until the outlet pockets, when leaves the computational domain.

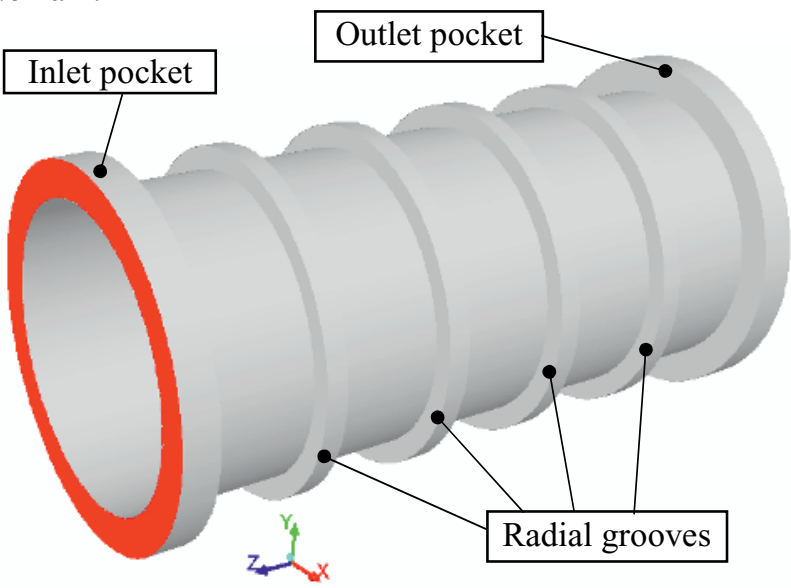

Figure 3. 3D model of the computational domain

\section{Computational grid, definition of the mathematical model of flow and boundary conditions}

The creation of 3D geometry of the computational domain was used DesignModeler software, which is part of the software ANSYS Fluent14.0. The final 3D model is illustrated in Figure 3. With regard to the rotation $\omega$ and precession $\Omega$ movement of the inner cylinder (Figure 4, Figure 1) is problem of defining the movement more difficult. Detailed description defining the movement is in [2]. Thus, it is necessary to consider changing the grid at each iteration step. Therefore it was selected from various alternatives from slipping grid where each of cylinder was surrounded by a layer of cells moving with and between these layers were formed annulus, which deforms see Figure 4.

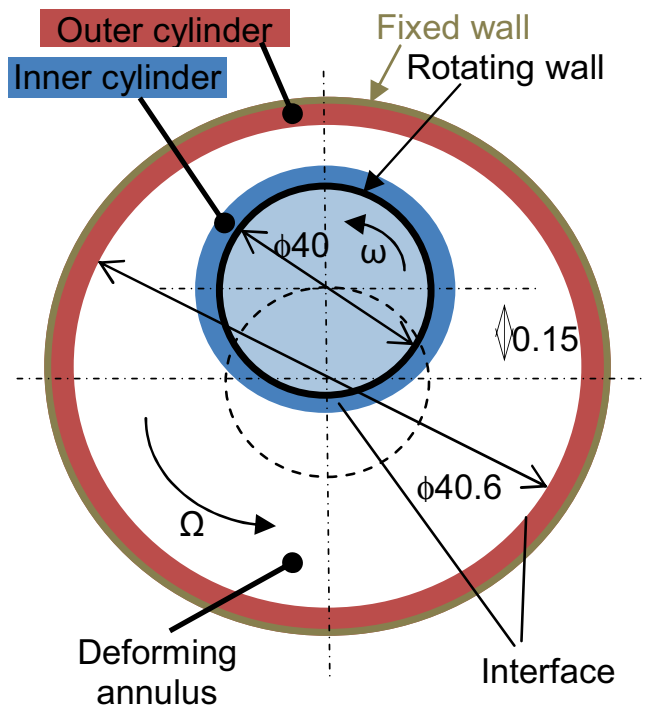

Figure 4. Description of the problem of rotation, the computational domain
The follow definition of problem movement areas when defining the mathematical model it was necessary to create 3D geometry as a set of three elements (cylinders), as marked in Figure 4 (the inner cylinder, deforming the annulus and the outer cylinder). Each area was then appropriately gridding with the corresponding number of cells along the height in the each area with function of the "Sweep" (sweeping surface mesh into space with a defined step). The total number of elements in the computing grid is 1068750 . View at the surface grid of the inner cylinder (rotor) is showed in Figure 5.

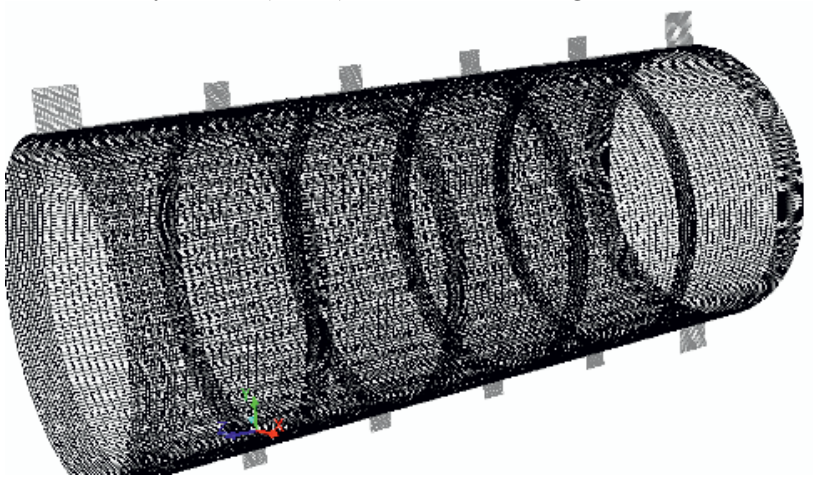

Figure 5. Computational grid on the surface of the rotor and in radial grooves

Detail view of computing grid in different areas including radial groove is shown in Figure 6. In the each area (the inner cylinder, deforming the annulus and the outer cylinder) are generated five layers of computing grid over the height, as shown in Figure 6. The radial grooves contained 20 layers of elements along the height.

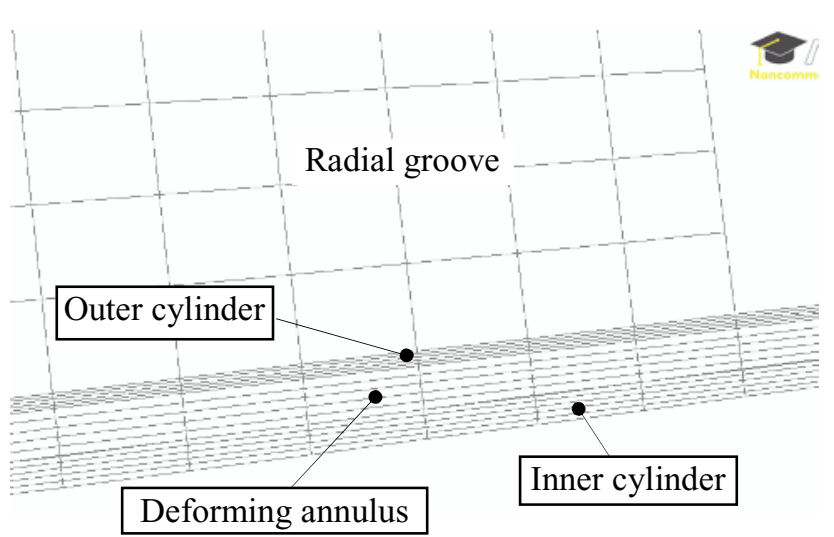

Figure 6. Detail of computer grid in the radial groove and in the narrow gap

\subsection{Mathematical model of flow}

Considering of the character problem is defined the threedimensional, time-dependent isothermal flow as incompressible. The flowing medium is water on the following defined physical properties. Based on the calculation of the Reynolds number for boundary conditions is defined laminar flow type. A detailed calculation and analysis of the Reynolds number is given in the [2-4].

Thus, the mathematical model of the flow is determined by the equation of continuity and Navier - Stokes 
equations, which is considered the laminar flow. The mathematical model is then solved in software ANSYS Fluent14.0 [4].

Equation of continuity

$$
\frac{\partial}{\partial t}+\frac{\partial\left(u_{j}\right)}{\partial x_{j}}=0
$$

Navier-Stokes equation:

$$
\begin{aligned}
& \frac{\partial\left(u_{i}\right)}{\partial t}+\frac{\partial\left(u_{i} u_{j}\right)}{\partial x_{j}}=-\frac{1}{\rho} \frac{\partial p}{\partial x_{i}}+\frac{\partial}{\partial x_{j}}\left(\mu \frac{\partial u_{i}}{\partial x_{j}}\right)+ \\
& +\delta_{i 3} g+f_{c} \varepsilon_{i j 3} u_{j}+f_{i}
\end{aligned}
$$

Flowing medium is water, which is defined by a constant value of the density $\rho=1000 \mathrm{~kg} \mathrm{~m}^{-3}$ and a kinematic viscosity $v=10^{-6} \mathrm{~m}^{2} \mathrm{~s}^{-1}$, thus defined incompressible flow.

\subsection{Boundary conditions}

The boundary conditions are defined at the inlet to the computational domain, the outlet from the domain and on the inner cylinder. The inlet and outlet boundary conditions are type of pressure. For inner cylinder (rotor) is defined rotation speed $\omega$ and speed precession $\Omega$ in

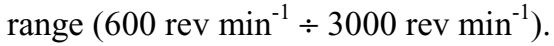

Parameters of the boundary conditions for numerical simulation:

- Inlet pressure (pressure condition, $p_{\text {rel }}=1.10^{5} \mathrm{~Pa}$ )

- Inlet pressure (pressure condition, $p_{\text {rel }}=0 \mathrm{~Pa}$ )

- Operation pressure $\left(p_{o}=101325 \mathrm{~Pa}\right)$

Table 1. Speed of rotation and precession

\begin{tabular}{|c|c|}
\hline Rotation & Precession \\
\hline$\omega=62.8319 \mathrm{rad} / \mathrm{s}$ & $\Omega=62.8319 \mathrm{rad} / \mathrm{s}$ \\
\hline$\omega=125.6637 \mathrm{rad} / \mathrm{s}$ & $\Omega=125.6637 \mathrm{rad} / \mathrm{s}$ \\
\hline$\omega=188.4956 \mathrm{rad} / \mathrm{s}$ & $\Omega=188.4956 \mathrm{rad} / \mathrm{s}$ \\
\hline$\omega=251.3274 \mathrm{rad} / \mathrm{s}$ & $\Omega=251.3274 \mathrm{rad} / \mathrm{s}$ \\
\hline$\omega=314.1593 \mathrm{rad} / \mathrm{s}$ & $\Omega=314.1593 \mathrm{rad} / \mathrm{s}$ \\
\hline
\end{tabular}

Total of 25 variants solutions were defined (all combinations of rotation $\omega$ and precession $\Omega$ of the rotor). The variants were solved as a time-dependent manner with a defined time step. The total computing time corresponded to two speed of rotor about the axis of the computational domain (two precession movements). The numerical calculation was an evaluation of the radial force $F_{r}$ and tangential force $F_{t}$ acting on the rotor in relation to time or rotating rotor. The next stage was evaluated pressure drop across the radial grooves for the various parameters of rotation and precession. To evaluate the radial and tangential forces acting on the rotor in the ANSYS Fluent14.0 first evaluated the force components $F_{x}$ and $F_{y}$ (force components in the coordinate system $x$ and $y$ axis). Subsequently, based on the conversion relationships can be determined by forces $F_{r}, F_{t}$ acting on the rotor. The conversion relationships are defined in [2].

\section{Evaluation of numerical simulation of 3D model of the flow in the narrow gap with radial grooves}

\subsection{Evaluation of forces $F_{r}$ a $F_{t}$ acting on the rotor}

First, for each variant boundary conditions (rotation and precession), see Table 1 were evaluated courses of forces $F_{x}$ and $F_{y}$ for two rotor speeds with the frequency of rotation of the rotor corresponding to the time step $\Delta t=0.0001 \mathrm{~s}$. Samples evaluated course of the forces $F_{x}$ and $F_{y}$ depending on the rotation for variant with a constant rotation of the rotor $\omega=62.8319 \mathrm{rad} \cdot \mathrm{s}^{-1}$ and different speeds of precession $\Omega$ are shown in Figure 7 and Figure 8.

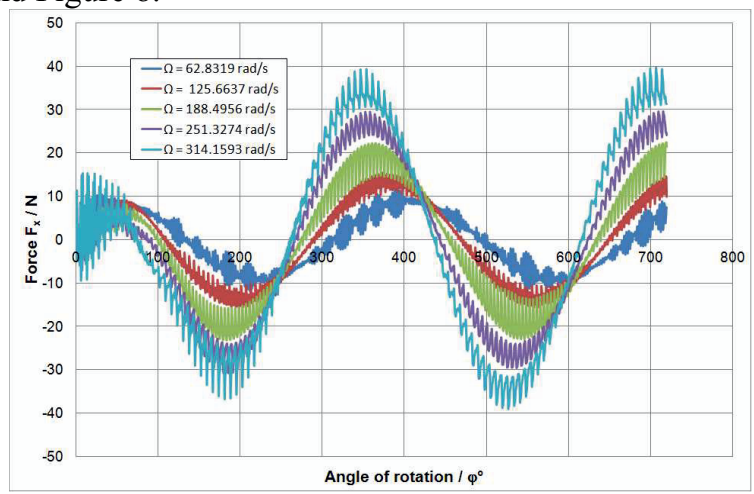

Figure 7. Distribution of $F_{x}$ depending on the angle of rotation $\varphi$ for constant rotation $\omega=62.8319 \mathrm{rad} \cdot \mathrm{s}^{-1}$ and variable precession $\Omega$

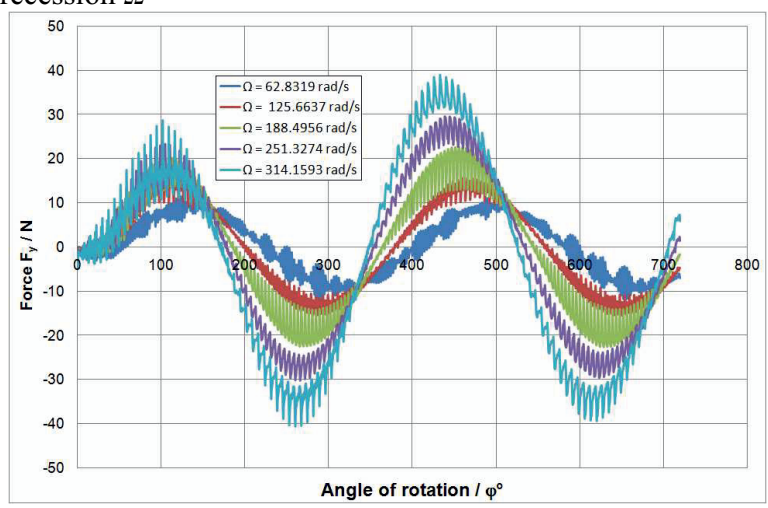

Figure 8. Distribution of $F_{y}$ depending on the angle of rotation $\varphi$ for constant rotation $\omega=62.8319 \mathrm{rad} \cdot \mathrm{s}^{-1}$ and variable precession $\Omega$

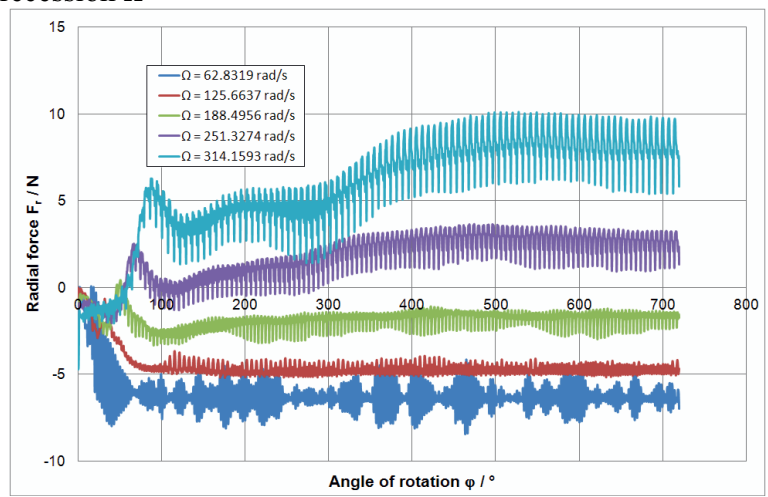

Figure 9. Distribution of radial force $F_{r}$ depending on the angle of rotation $\varphi$ for constant rotation $\omega=62.8319 \mathrm{rad} \cdot \mathrm{s}^{-1}$ and variable precession $\Omega$ 


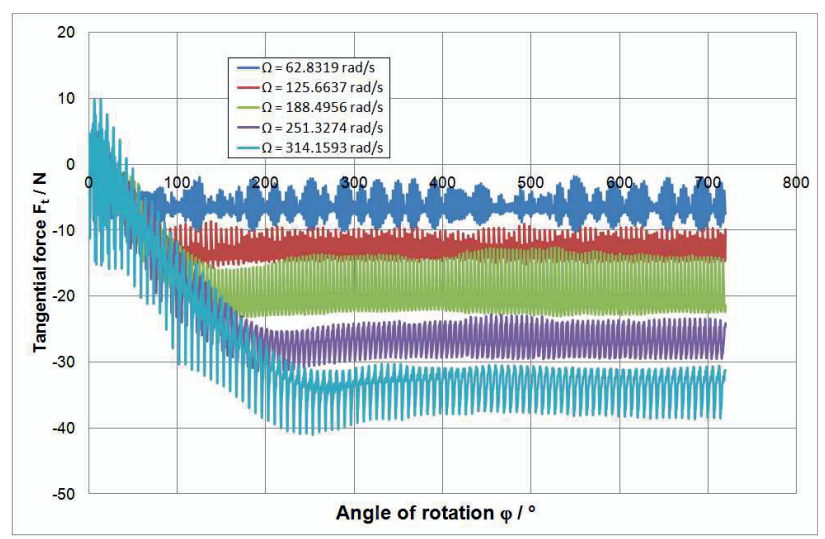

Figure 10. Distribution of tangential force $F_{t}$ depending on the angle of rotation $\varphi$ for constant rotation $\omega=62.8319 \mathrm{rad} \cdot \mathrm{s}^{-1}$ and variable precession $\Omega$

Subsequently, the component forces are converted to radial force $F_{r}$ and tangential $F_{t}$ and graphically plotted their course (Figure 9, Figure 10). In the same method were evaluated the courses of $F_{x}, F_{y}, F_{r}$ and $F_{t}$ for all combinations of rotation $\omega$ and precession $\Omega$ of rotor, which are specified in the boundary conditions. Subsequently from the graphic dependence were evaluated the mean values of radial and tangential force $F_{r}$ and $F_{t}$.

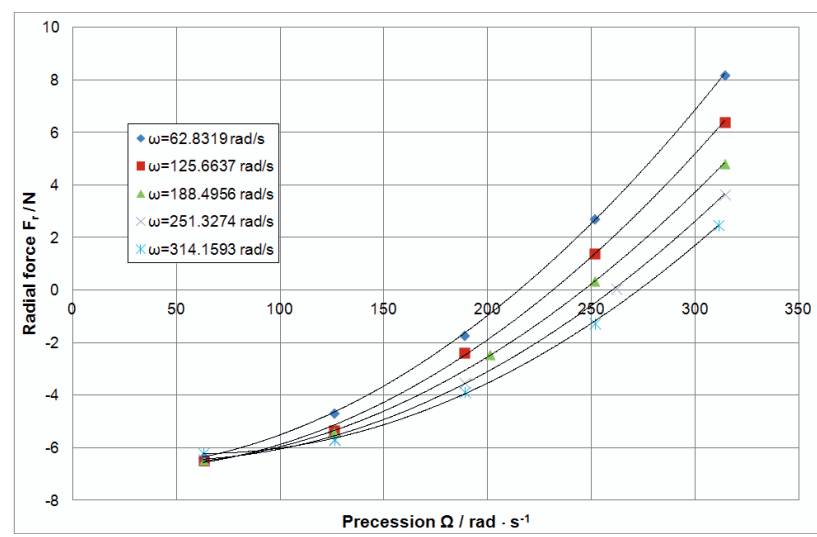

Figure 11. Distribution of radial force $F_{r}$ depending on the precession $\Omega$ for constant rotation $\omega$

From the evaluation of functional dependence (figureure 11) were determined polynomial regression dependencies:

$$
F_{r}(\omega)=A+B \cdot \Omega+C \cdot \Omega^{2}
$$

Therefore

$$
\begin{aligned}
& F_{r(\omega=62.8319 \mathrm{rad} / \mathrm{s})}=1.645274 \cdot 10^{-4} \Omega^{2}-3.925143 \cdot 10^{-3} \Omega-6.764612 \\
& F_{r(\omega=125.6637 \mathrm{rad} / \mathrm{s})}=1.547658 \cdot 10^{-4} \Omega^{2}-6.547834 \cdot 10^{-3} \Omega-6.77141 \\
& F_{r(\omega=188.4956 \mathrm{rad} / \mathrm{s})}=1.425181 \cdot 10^{-4} \Omega^{2}-8.539407 \cdot 10^{-3} \Omega-6.536898 \\
& F_{r(\omega=251.3274 \mathrm{rad} / \mathrm{s})}=1.389478 \cdot 10^{-4} \Omega^{2}-1.220619 \cdot 10^{-2} \Omega-6.222447 \\
& F_{r(\omega=314.1593 \mathrm{rad} / \mathrm{s})}=1.388646 \cdot 10^{-4} \Omega^{2}-1.693195 \cdot 10^{-2} \Omega-5.713129
\end{aligned}
$$

In Table 2 are summarized the values of the coefficients of the polynomial relations $A, B$ and $C$, which may draw in dependence to the rotation $\omega$ (Figure 12, Figure 13, Figure 14).
Table 2. Evaluation of the coefficients A, B, C

\begin{tabular}{|c|c|c|c|}
\cline { 2 - 4 } \multicolumn{1}{c|}{} & $\boldsymbol{A}$ & $\boldsymbol{B}$ & $\boldsymbol{C}$ \\
\hline$[\omega]=62.8319 \mathrm{rad} / \mathrm{s}$ & -6.7646 & -0.00392514 & $1.645 \mathrm{E}-04$ \\
\hline$[\omega]=125.6637 \mathrm{rad} / \mathrm{s}$ & -6.771 & -0.00654783 & $1.548 \mathrm{E}-04$ \\
\hline$[\omega]=188.4956 \mathrm{rad} / \mathrm{s}$ & -6.5369 & -0.00853941 & $1.425 \mathrm{E}-04$ \\
\hline$[\omega]=251.3274 \mathrm{rad} / \mathrm{s}$ & -6.2224 & -0.0122062 & $1.389 \mathrm{E}-04$ \\
\hline$[\omega]=314.1592 \mathrm{rad} / \mathrm{s}$ & -5.7131 & -0.0169319 & $1.389 \mathrm{E}-04$ \\
\hline
\end{tabular}

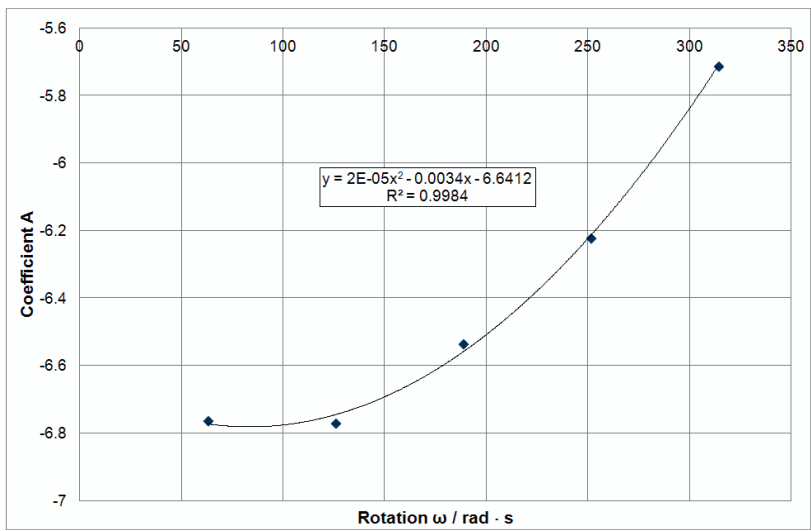

Figure 12. Course of the coefficient $A$ in depending on the rotation $[\omega]=\mathrm{rad} \cdot \mathrm{s}^{-1}$

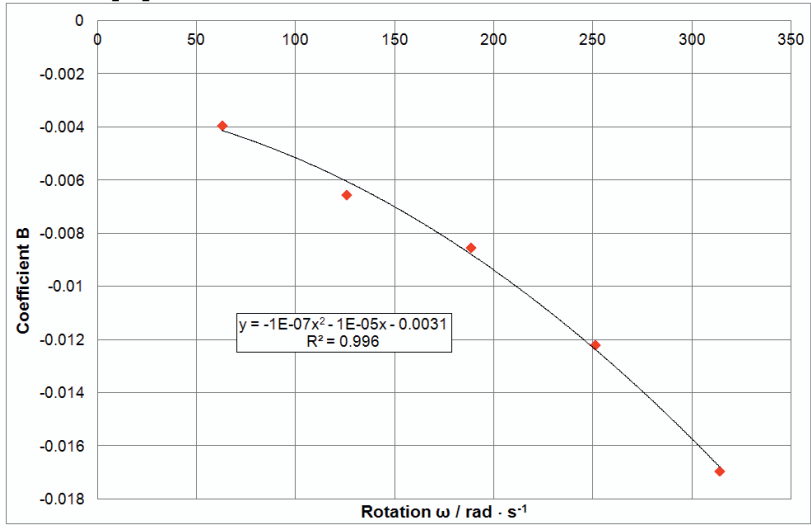

Figure 13. Course of the coefficient $B$ in depending on the rotation $[\omega]=\mathrm{rad} \cdot \mathrm{s}^{-1}$

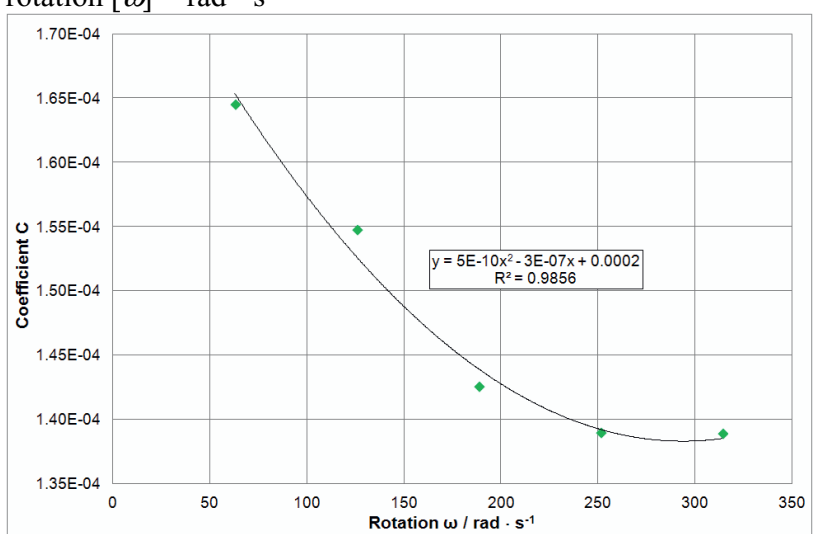

Figure 14. Course of the coefficient $B$ in depending on the rotation $[\omega]=\operatorname{rad} \cdot \mathrm{s}^{-1}$ 


\subsection{Evaluation of the pressure drop between the radial grooves}

Further evaluation of numerical simulation is focused at the evaluation the pressure gradient $\Delta p_{i}$ at various radial grooves, as shown in Figure 15.

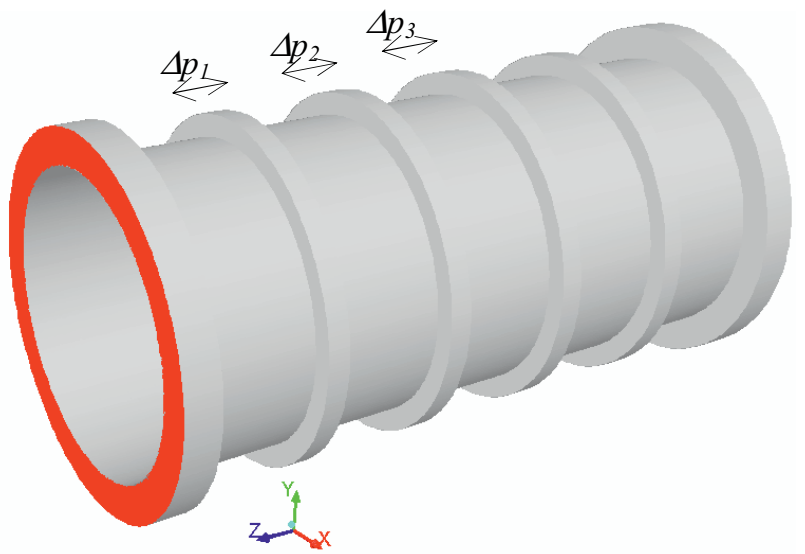

Figure 15. Designation evaluated pressure drops $\Delta p_{i}$ on the individual grooves

Evaluation of pressure drop at the each radial grooves is graphically shown for a constant rotation of the rotor $\omega$ depending on the precession motion $\Omega$ (Figure 16, Figure 17, Figure 18, Figure 19, Figure 20).

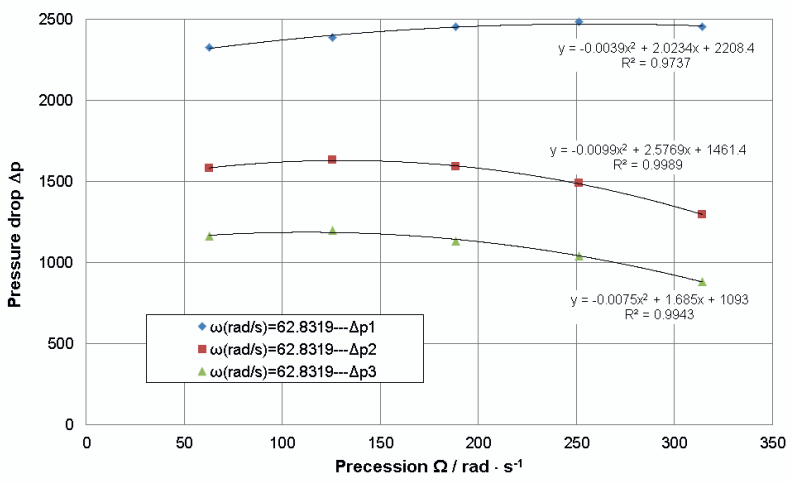

Figure 16. The dependence of pressure drop at each radial grooves $\left(\Delta p_{1}, \Delta p_{2}, \Delta p_{3}\right)$ for constant rotation $\omega=62.8319 \mathrm{rad} \cdot \mathrm{s}^{-1}$ and variable precession $\Omega$

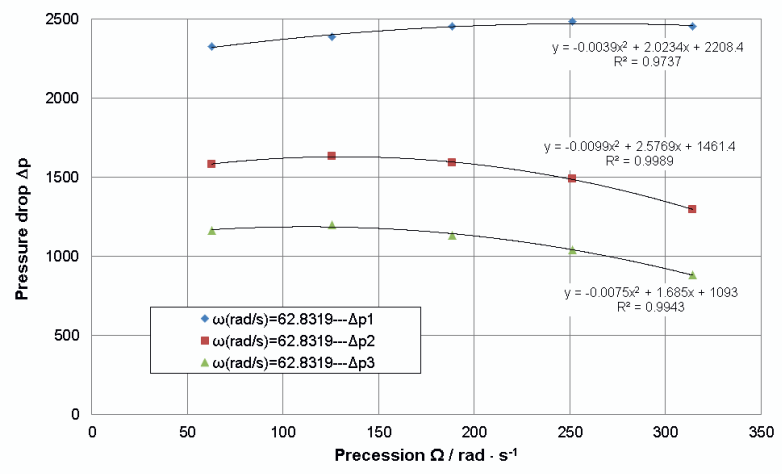

Figure 17. The dependence of pressure drop at each radial grooves $\left(\Delta p_{1}, \Delta p_{2}, \Delta p_{3}\right)$ for constant rotation $\omega=125.6637 \mathrm{rad}$. $\mathrm{s}^{-1}$ and variable precession $\Omega$

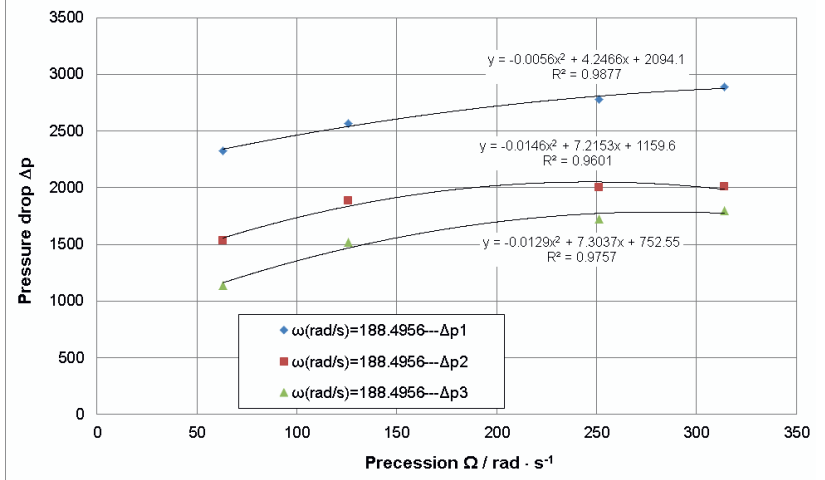

Figure 18. The dependence of pressure drop at each radial grooves $\left(\Delta p_{1}, \Delta p_{2}, \Delta p_{3}\right)$ for constant rotation $\omega=188.4956 \mathrm{rad}$. $\mathrm{s}^{-1}$ and variable precession $\Omega$

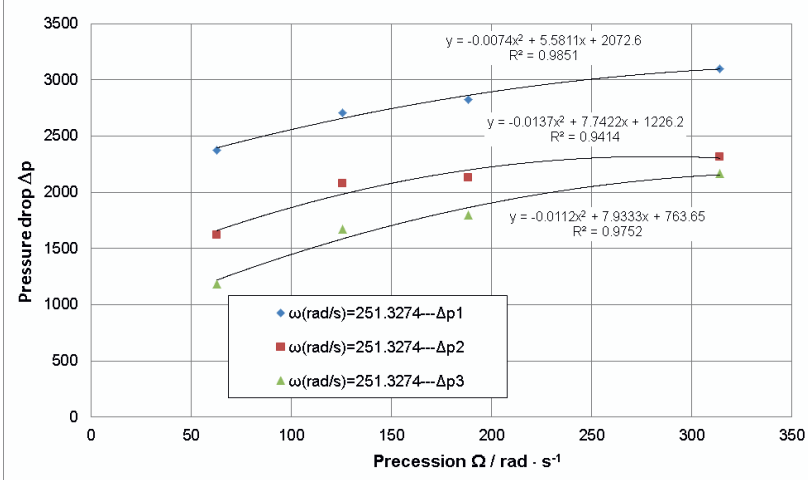

Figure 19. The dependence of pressure drop at each radial grooves $\left(\Delta p_{1}, \Delta p_{2}, \Delta p_{3}\right)$ for constant rotation $\omega=251.3274 \mathrm{rad} \cdot$ $\mathrm{s}^{-1}$ and variable precession $\Omega$

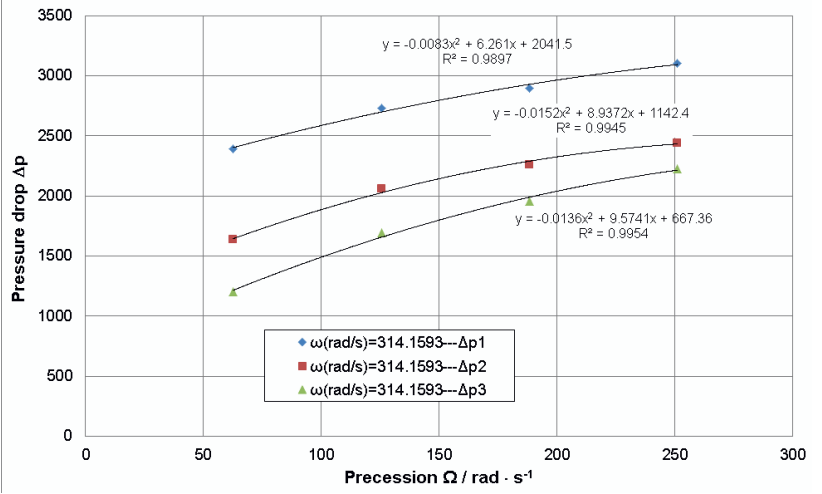

Figure 20. The dependence of pressure drop at each radial grooves $\left(\Delta p_{1}, \Delta p_{2}, \Delta p_{3}\right)$ for constant rotation $\omega=314.1592 \mathrm{rad}$. $\mathrm{s}^{-1}$ and variable precession $\Omega$

From the obtained results show that the maximum pressure drop is at the first groove $\Delta p_{l}$, and gradually decreases the further grooves. Subsequently, it is apparent that with the rising speed of precessional motion $\Omega$ increased of pressure drop. Next evaluation represents the course of the pressure drop $\Delta p$ in individual radial grooves in dependence on the rotation $\omega$ and precession $\Omega$ of the rotor. 


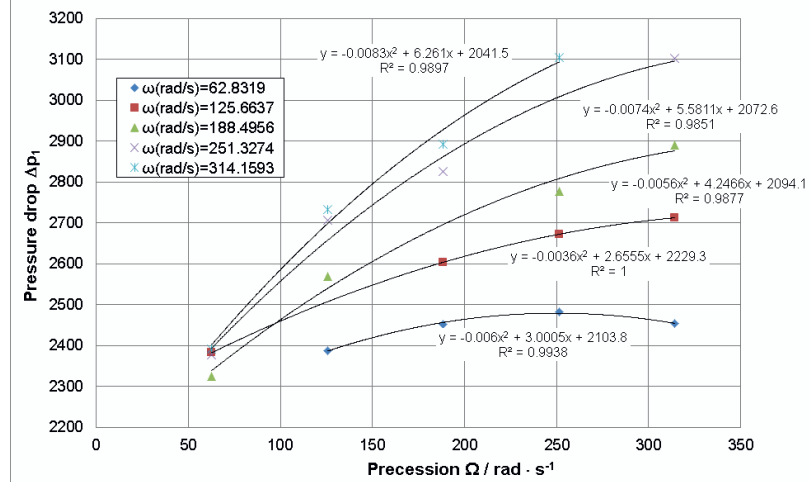

Figure 21. The dependence of pressure drop at the first radial grooves $\Delta \mathrm{p}_{1}$ for variable precession $\Omega$ and rotation $\omega$

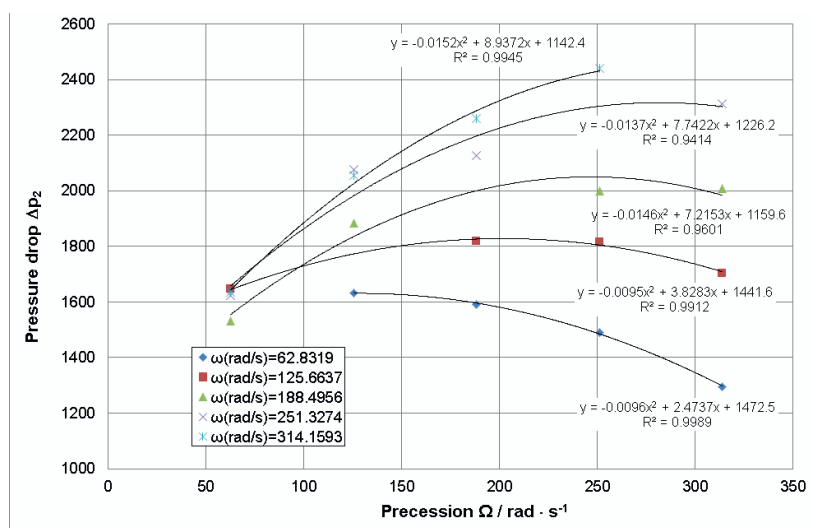

Figure 22. The dependence of pressure drop at the second radial grooves $\Delta \mathrm{p}_{1}$ for variable precession $\Omega$ and rotation $\omega$

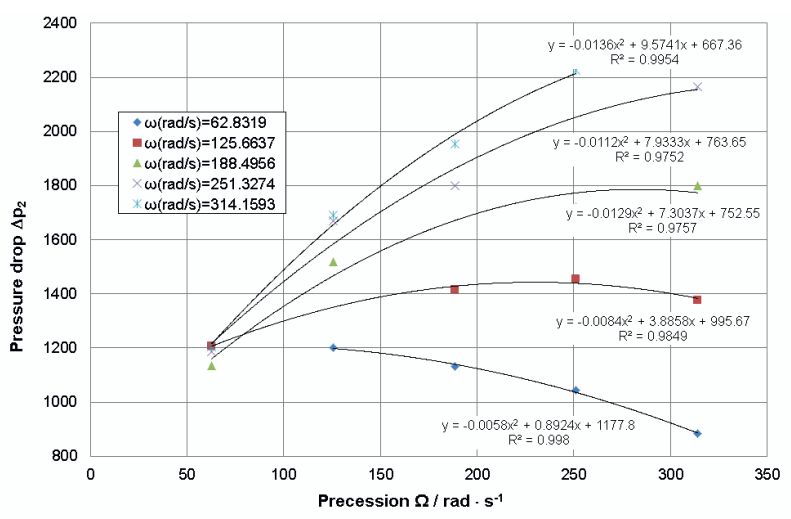

Figure 23. The dependence of pressure drop at the third radial grooves $\Delta \mathrm{p}_{1}$ for variable precession $\Omega$ and rotation $\omega$

On Figure 21, Figure 22 and Figure 23 is a noticeable growth of pressure drop $\Delta p$ in dependence to the increasing speed of rotation $\omega$ and $\Omega$ precession.

\section{Conclusion}

The article defines the problem of mathematical modeling of fluid flow in the narrow gap with radial grooves between the two cylinders, when the inner cylinder is eccentrically deposited and held simultaneously rotating and precessing circular motion. In the first phase by numerical simulations are evaluated radial and tangential forces $\left(F_{r}\right.$ and $\left.F_{t}\right)$ acting on the inner rotor. In the next phase the pressure drops $\Delta \mathrm{p}_{\mathrm{i}}$ is evaluated at each radial grooves. Thus defined approaches to solution of the flow in the narrow gap with consideration of motion can be applied in many engineering. The advantage of the mathematical modeling in software ANSYS Fluent is that a simple modification of geometry, in this case, for example, size or shape of the radial grooves can evaluate how it will affect the size of the pressure drop and the size of the forces, and thereby optimize the shape of the grooves. Other applications, which can be used as follows defined methodology as in the case of hydrostatic lubrication of bearings, flow modeling sealing gaps pumps or turbines and in many other technical applications.

\section{Acknowledgments}

Work was supported by specific research SP2012/55 (Modeling the dynamics of fluid elements and systems) and GAP101/10/0209 (Research and modeling of flow control limits magnetorheological fluid in a thin lubricating film on the rotor vibration)

\section{Reference}

1. F. Pochylý, S. Fialová, M. Kozubková, M. Bojko. 26th IAHR Symposium on Hydraulic Machinery and Systems. 15, (2012).

2. M. Bojko, M. Kozdera, M. Kozubková. EPJ Web of Conferences. 45, 6 (2013).

3. J. Jablonská. Modelling on cavitation in a diffuser with vortex generator. 45, 6 (2013).

4. M. Kozubková. Numerické modelování proudění FLUENT I. 2008.

5. ANSYS Fluent 14 - User's Guide. Fluent Inc, 2011.

6. R. B. Bird, W. E. Stewart, E. N. Lightfoot. Transport Phenomena. John Wiley \& Sons. 895 p. 2001.

7. H. Black, J. Mech. Eng. Scien. 11, 8 (1969). 